Second Day: Tue., Nov. 8

Morning Session Short-Range Forecast II (Basic research)

A New Atmospheric Model for Numerical Prediction

A. Eliassen

Integration of Balanced Equation

R. FJфRTOFT

Calculations for the Hemisphere with the Barotropic Primitive Equations

\title{
N. Phillips
}

The hemispheric computation net described by the author in the 75 th Anniversary Volume of the Meteorological Society of Japan has been tested on two sets of idealized initial data. The so-called primitive equations for a barotropic atmosphere were used in these preliminary tests. Best results are obtained when the Eliassen finite-difference representation is used and the individual time derivative is evaluated as a centered difference and as an uncentered difference at alternate time steps.

\section{Numerical Experiments with the Primitive Equations}

\section{F. G. Shuman}

Current results from research in progress will be presented.

\section{Non-Geostrophic Effects in the Baroclinic Prognostic Equation}

\section{A. Arakawa}

Some results of a preliminary study to introduce the non-geostrophic effects into the baroclinic prognostic equations are presented. From the energetical point of view, severa] balanced non-geostrophic models are proved to be consistent, but they clearly have different functions to constrain the behavior of atmospheric motion. In a simple baroclinic case, some of these functions especially for the development and movement of disturbance are compared with those of quasi-geostrophic model.

Problems on numerical treatment of non-geostrophic effects also are discussed. 